\title{
How much can you "enhance" recovery after lung resection?
}

Eric Lim, MB, ChB, MD, MSc, FRCS(C-Th)

\author{
From the Academic Division of Thoracic Surgery, The Royal Brompton Hospital, London, United Kingdom. \\ Dr Lim reports personal fees from Abbott Molecular, Glaxo Smith Kline, Pfizer, Norvatis, Covidien, Roche, Lily \\ Oncology, Boehringer Ingelheim, Medela, Ethicon, and grants and personal fees from ScreenCell. \\ Received for publication Nov 2, 2017; accepted for publication Nov 9, 2017; available ahead of print Jan 13, 2018. \\ Address for reprints: Eric Lim, MB, ChB, MD, MSc, FRCS(C-Th), Academic Division of Thoracic Surgery, The \\ Royal Brompton Hospital, Sydney St, London SW3 6NP, United Kingdom (E-mail: e.lim@rbht.nhs.uk). \\ J Thorac Cardiovasc Surg 2018;155:1853-4 \\ $0022-5223 / \$ 36.00$ \\ Copyright (C) 2017 by The American Association for Thoracic Surgery \\ https://doi.org/10.1016/j.jtcvs.2017.11.016
}

The enhanced recovery after surgery movement is certainly gathering momentum. Origins can be traced as far back as the 1990s in colorectal surgery, where fast-track programs achieved a median postoperative hospital stay of 2 days in patients undergoing open colectomy compared with the accepted postoperative hospital stay of 5 to 10 days at the time. ${ }^{1}$ Since then, "enhanced recovery" (a more politically correct-sounding phrase compared with "fast track") has been promoted across many Health Boards in Europe and the United Kingdom (National Health Service). The concept is laudable and straightforward: to optimize all aspects of multidisciplinary patient care from referral, assessment, admission, to follow-up to ensure patients recover faster and better, leading to a reduction in length of stay.

In this issue of the Journal, Rogers and colleagues ${ }^{2}$ from Bristol, United Kingdom, take this one step further and report that enhanced recovery reduced morbidity in a cohort study of 422 patients who underwent lung cancer resection, with a median hospital stay of 5 days. However, the conclusions were based on the ability of the completion of all components of the enhanced recovery program, and they were not able to differentiate between cause and effect, that is, whether the onset of complications resulted in the inability to complete the components of the pathways (such as walking within 24 hours) rather than vice versa.

Data on the efficacy of enhanced recovery in thoracic surgery are not consistent. To be featured shortly in the Journal is a report by Brunelli and colleagues ${ }^{3}$ that the introduction of an enhanced recovery program in Leeds, United Kingdom, did not lead to reduction in complications nor length of stay (median of 5 days). Of note, at the Royal Brompton Hospital, which does not presently have an enhanced recovery program, the audited median length of stay for patients undergoing lobectomy for primary lung cancer for the fiscal year 2015/2016 was also 5 days.

That is not to say that the introduction of an enhanced recovery pathway is unwarranted. Any improvement to the processes of patient preparation, procedure, and in-patient care should be the aim of all institutions and does not need validation in a randomized trial. However, proponents of the enhanced recovery program need to be cautious of

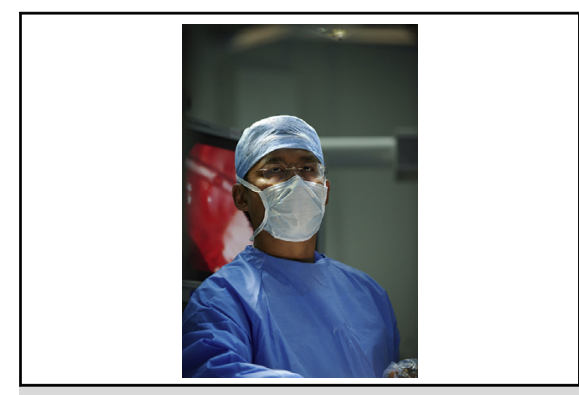

Eric Lim, MB, ChB, MD, MSc, FRCS(C-Th), consultant thoracic surgeon.

\section{Central Message}

Enhanced recovery after surgery is an important movement but has limited demonstration of efficacy. A customized thoracic surgery model is required to achieve the aims of optimizing recovery after lung resection.

See Article page 1843.

their claims on efficacy, which are mainly based in colorectal surgery rather than thoracic surgery.

We have a long way to go from a median of 5 days stay for lung cancer resection, and it is the aim of my team to get patients ready for home within 2 days, whether it is a single-port video-assisted thoracoscopic surgery or an open lung resection, through optimizing clinical care protocols. Although components of an enhanced recovery are important, a detailed analysis identified the duration of chest drain as the single most important determinant of length of stay, ${ }^{4}$ as surgeons usually send patients home only after the drain has been removed. We identified that fluid volume cut-off is the bottle neck that determines the duration of chest drain. If no restrictions are placed on fluid volume, then the majority of drains are removed on the first postoperative day. The efficacy of the individual components of an enhanced recovery program cannot improve on length of stay without first freedom from the drain.

I am strongly supportive of enhanced recovery program, but I feel that it needs to be customized to thoracic surgery rather than adopted from colorectal surgery. We are at the start where colorectal surgeons began trying to improve recovery to reduce length of stay from 5 to 2 days. This will also be achievable by focusing on issues pertinent to thoracic surgery, such as appropriate and judicious use of central lines, urinary catheters, oxygen therapy (all of which inhibit mobility), better pain control (with intraoperative local anesthesia), and optimizing drain management (with digital drains) in 
conjunction with improvement in process of care in a customized thoracic surgery-enhanced recovery program.

\section{References}

1. Kehlet H. Multimodal approach to control postoperative pathophysiology and rehabilitation (review). Br J Anaesth. 1997;78:606-17.

2. Rogers LJ, Bleetman D, Messenger DE, Joshi NA, Wood L, Rasburn NJ, et al. The impact of enhanced recovery after surgery (ERAS) protocol compliance on morbidity from resection for primary lung cancer. J Thorac Cardiovasc Surg. 2018; 155:1843-52.

3. Brunelli A, Thomas C, Dinesh P, Lumb A. Enhanced recovery pathway versus standard care in patients undergoing video-assisted thoracoscopic lobectomy. J Thorac Cardiovasc Surg. 2017;154:2084-90.

4. Mesa-Guzman M, Periklis P, Niwaz Z, Socci L, Raubenheimer H, Adams B, et al. Determining optimal fluid and air leak cut off values for chest drain management in general thoracic surgery. $J$ Thorac Dis. 2015;7:2053-7. 